\title{
An Optimization Framework for Resource Allocation in Virtual Sensor Networks
}

\author{
Carmen Delgado*, José Ramón Gállego*, María Canales*, Jorge Ortín*†, Sonda Bousnina ${ }^{\ddagger}$, Matteo Cesana ${ }^{\ddagger}$ \\ *Aragón Institute of Engineering Research ${ }^{\dagger}$ Centro Universitario de la Defensa Zaragoza \\ Universidad de Zaragoza, \\ Zaragoza, Spain \\ Academia General Militar, \\ Zaragoza, Spain \\ \{cdelga, jrgalleg, mcanales\}@unizar.es_ jortin@unizar.es \\ $\ddagger$ Dipartimento di Elettronica, \\ Informazione e Bioingegneria, \\ Politecnico di Milano, \\ Milano, Italy \\ \{sonda.bousnina, matteo.cesana\}@polimi.it
}

\begin{abstract}
We propose an optimization framework to perform resource allocation in virtual sensor networks. Sensor network virtualization is a promising paradigm to improve flexibility of wireless sensor networks which allows to dynamically assign physical resources to multiple stakeholder applications. The proposed optimization framework aims at maximizing the total number of applications which can share a common physical network, while accounting for the distinguishing characteristics and limitations of the wireless sensor environment (limited storage, limited processing power, limited bandwidth, tight energy consumption requirements). The proposed framework is finally applied to realistic network topologies to assess the gain involved in letting multiple applications share a common physical network with respect to one-application, one-network vertical design approaches.
\end{abstract}

\section{INTRODUCTION}

Wireless Sensor Networks (WSNs) constitute one of the main building blocks to realize the vision of the Internet of Things (IoT), where huge numbers of smart objects interacting with the surrounding environment have a "presence" on the Internet. WSNs are used nowadays to support many applications/services in diverse scenarios and environments ranging from smart home or environmental monitoring based on scalar sensed data to more demanding applications based on multimedia sensors.

Usually, WSNs are designed and deployed in a "vertical", application-specific way, in which the hardware and network resources are customized to the specific application requirements. On one hand, such design paradigm allows to have "optimal" performance on the specific application, but, on the other hand, it precludes resources (hardware and software) reuse when other applications and services must be contemplated. In the end, this has led in the past to the proliferation of redundant WSNs deployments [1].

In this context, virtualization is a promising technique to target more efficient resource utilization, lower cost and increased flexibility and manageability in WSN deployments [2]. The key idea behind virtualization is to decouple the physical infrastructure and resources from application ownerships. Thus, network virtualization technologies abstract away "physical resources" including node processing/storage capabilities, available communication bandwidth and routing protocols, which can then be "composed" at a logical level to support usage by multiple independent users and even by multiple concurrent applications [3]. While network virtualization is already a reality in many communication networks [4], [5], research on sensor network virtualization is still in its infancy and comprehensive solutions still need to be found to cope with the specific characteristics of WSNs in terms of limited node capabilities and communication bandwidth [6].

In this work, we focus on the design of a virtualization engine for WSNs. Namely, we consider a general purpose WSN which can be used to support multiple applications and we propose a mathematical programming framework to optimally allocate virtual sensor networks to the requesting applications. The proposed framework allocates the physical resources of the general purpose WSN to multiple concurrent application while accounting for the limitations in the physical resources (processing, storage, available bandwidth, limited communication range) and the specific application requirements. Numerical results are then obtained by applying the proposed framework to realistic WSN instances to assess the efficiency of the virtualization process.

The paper is organized as follows: Section II overviews the related work in the field of sensor network virtualization. Section III describes the proposed system model and the optimization problem for resource allocation in virtual sensor networks. In Section IV, the proposed model is evaluated by simulation for a set of scalar and multimedia applications also with different types of sensor nodes. Finally, some conclusions are provided in Section V.

\section{RELATED WORK}

The emergence of shared sensor networks has stimulated research efforts in the field of novel programming abstractions at the node level and management framework at the network level to support multiple applications over a shared physical infrastructure [7], [8].

At the node level, architectures based on virtual machines are proposed to enable virtualization and re-programmability. As an example, Mate [9], ASVM [10], Melete [11] and VMStar [12] are frameworks for building application-specific virtual machines over constrained sensor platforms. 
At the network level, several virtualization management platforms have been introduced. SenSHare [13] creates multiple overlay sensor networks which are "owned" by different applications on top of a shared physical infrastructure. UMADE [14] is an integrated system for allocating and deploying applications in shared sensor networks based on the concept of Quality of Monitoring (QoM). Fok et al. [15] introduce middleware abstractions to represent multiple QoM requirements from multiple applications, whereas a service-oriented middleware is presented in [16] to address the challenges faced by running multiple applications onto heterogeneous WSNs.

Generally speaking, the aforementioned work provides "practical" building blocks to build up virtual sensor networks. Differently, we focus in this paper on the "intelligence" to properly allocate physical resources to virtual applications, which can be cast as a general resource allocation problem. Even if radio/network resource allocation is a widely debated topic in the literature, still very few works have appeared on the optimal resource allocation in the field of Virtual or Shared Sensor Networks.

In [17] the authors propose an optimization framework to maximize the Quality of Monitoring (QoM) in shared sensor networks. The proposed framework focuses on environmental monitoring applications whose reference "quality" can be modeled as dependent on the variance in the sensed data, and derives the application-to-sensors assignment which minimizes such variance. The same authors address in a later work the case where the application assignment problem is no longer centralized but rather distributed by resorting to game-theoretic tools [18]. Ajmal et al. leverage the concept of QoM and propose an admission control scheme to dynamically "admit" applications to be deployed on physical sensor networks. The authors of [19] focus on the problem of scheduling applications to shared sensor nodes with the ultimate goal of maximizing the sensor network lifetime.

The problem of allocating physical resources to multiple application can be also cast as an auction. In [20], the authors propose a reverse combinatorial auction, in which the sensor nodes act as bidders and bid cost values (according to their available resources) for accomplishing the subset of the applications' tasks. Optimal bidding strategies are then studied to make the auction effective and truthful.

\section{SYSTEM MODEL AND OPTIMIZATION FRAMEWORK}

Let $S=\left\{s_{1}, s_{2}, \ldots, s_{l}\right\}$ be a set of sensor nodes, $A=$ $\left\{a_{1}, a_{2}, \ldots, a_{m}\right\}$ a set of applications which are to be deployed in the reference area, and $T=\left\{t_{1}, \ldots, t_{n}\right\}$ a set of test points in the reference network scenario. To simplify notation, in the following we will use the subscript index $i$ (or $h$ ) to refer to a sensor node $s_{i}$ (or $s_{h}$ ), the subscript index $j$ to refer to an application $a_{j}$ and the subscript index $k$ to refer to a test point $t_{k}$.

Each application $j$ requires to cover a given set of test points $T_{j} \subseteq T$. Formally, the application $j$ has to be deployed on a subset of $S$ such that all the test points in $T_{j}$ are covered. A test point is covered by a sensor node $i$ if it is within its sensing range, $R_{i}^{s}$. It is convenient to introduce as well the set $S_{j k}$ defined as the set of sensor nodes which physically cover the test point $k$, with $k \in T_{j}$. In other words, if the application $j$ is deployed on any of the sensors in set $S_{j k}$, then the target test point $k$ is covered for this application. A necessary condition for an application $j$ to be successfully deployed is that all the test points in its target set $T_{j}$ must be covered.

Each application $j$ in $A$ is further characterized by a requirement vector $r_{j}=\left\{c_{j}, m_{j}, l_{j}\right\}$ which specifies the required source rate [bit/s], memory [bits] and processing load [MIPS] consumed by the application when it is deployed on a sensor node. The requirement vector can be interpreted as the amount of resources needed to accomplish the specific tasks required by the application (e.g., acquire, process and transmit 10 temperature samples, or acquire process and transmit one JPEG image, etc.). Additionally, each sensor node $i$ in $S$ is characterized by a given resource vector $o_{i}=\left\{C_{i}, M_{i}, L_{i}, E_{i}\right\}$, which specifies its available bandwidth, storage capabilities, processing power and energy store.

A protocol interference model with power control [21] is used to characterize the wireless communications among the sensor nodes. The maximum transmission power is $P_{\max }$. With this power, there are a maximum transmission range $R_{\max }^{T}$ and a maximum interference range $R_{\max }^{I}$. Given a directional link between a pair of nodes $(i, h)$, the channel gain from transmitter $i$ to receiver $h$ is defined as $g_{i h}=g_{0} \cdot d_{i h}^{-\gamma}$, being $d_{i h}$ the distance from $i$ to $h, \gamma$ the path loss index and $g_{0}$ a constant dependent on antenna parameters. A transmission is successful if the received power exceeds a threshold $\alpha$. Additionally, all the nodes under the interference range of a sensor node share the same transmission channel and therefore, the transmission time must be divided between them. If $p_{i}$ is the transmission power assigned to node $i$, a transmission towards $h$ is successful if $p_{i} \cdot d_{i h}>\alpha$. Thus, the transmission range for node $i$ with transmission power $p_{i}$ can be obtained as $R_{i}^{T}\left(p_{i}\right)=\left(p_{i} \cdot g_{0} / \alpha\right)^{-\gamma}$. Similarly, the interference resulting from node $i$ with power $p_{i}$ is non-negligible only if it exceeds a certain threshold $\beta$. Then, the interference range is $R_{i}^{I}\left(p_{i}\right)=\left(p_{i} \cdot g_{0} / \beta\right)^{-\gamma}$.

Qualitatively, the application assignment problem for virtual sensor networks can be defined as follows: to maximize the weighted number of deployed applications subject to coverage constraints (the set of test points of each application must be covered) and application requirements (each application should be assigned enough bandwidth, and processing and storage resources to operate successfully). In addition, due to the multihop nature of WSNs, routing and link capacity constraints must be considered when the data generated by the application has to be delivered remotely.

Further, let us assume that a preference vector across all the $m$ applications is defined $Q=\left[q_{1}, q_{2}, \ldots, q_{m}\right]^{T}$ where $q_{j}$ represents the revenue for the network provider for having application $j$ successfully deployed in the network. Let $z_{j}$ be a binary variable indicating if application $j$ is successfully deployed in the network. Let $y_{i j k}$ be a binary variable indicating 
if test point $k$ of application $j$ is deployed at sensor node $i$. Let $x_{i}$ be a binary variable indicating if sensor node $i$ is active in the network. Let $h_{j k}$ be a binary variable which indicates if test point $k$ belonging to set $T_{j}$ is actually covered by a sensor node which runs application $j$.

The objective function aims at maximizing the overall revenue out of the application deployment process while minimizing the cost related to activating sensor nodes:

$$
\max \left(\sum_{j \in A} q_{j} z_{j}-\sum_{i \in S} \delta_{i} x_{i}\right)
$$

where $\delta_{i}$ is the cost incurred in activating sensor node $i$.

Constraints (2), (3) and (4) require that all the applications which are actually deployed do fulfill the coverage constraints, that is, they cover all the required test points. Specifically, Eq. (2) indicates if test point $k$ of an application $j$ is effectively covered. If so, it ensures that it is covered by only one sensor node $i$. Depending on the application, it can be possible that the same sensor node can cover several of its test points (e.g., visual applications). If we define $N_{i j}$ as the maximum number of test points of the same application $j$ that a sensor $i$ is able to cover, Eq. (3) guarantees that this threshold is not exceeded. Eq. (4) indicates that if an application $j$ is successfully deployed, i.e., if $z_{j}=1$, then all the test points of application $j$ must be covered. In addition, it guarantees that if the application cannot be deployed, none of its test points is covered so that no resources are wasted.

$$
\begin{gathered}
\sum_{i \in S_{j k}} y_{i j k}=h_{j k} \quad \forall j \in A, \forall k \in T_{j} \\
\sum_{k \in T_{j}} y_{j k} \leq N_{i j} \quad \forall i \in S, \forall j \in A \\
z_{j}=\frac{\sum_{k \in T_{j}} h_{j k}}{\left|T_{j}\right|} \quad \forall j \in A
\end{gathered}
$$

Constraints (5) and (6) are budget-type constraints for the available storage and processing load of the sensor nodes.

$$
\begin{gathered}
\sum_{j \in A} \sum_{k \in T_{j}} m_{j} y_{i j k} \leq M_{i} \quad \forall i \in S \\
\sum_{j \in A} \sum_{k \in T_{j}} l_{j} y_{i j k} \leq L_{i} \quad \forall i \in S
\end{gathered}
$$

The deployed applications will require most likely that the information generated locally is delivered remotely to collection points (sink nodes) through multihop paths. Note that these sensor nodes may run deployed applications or not. By resorting to a fluid model, it should be ensured that all the data produced by the sensors running applications is received by the sink nodes. This fact can be conveniently expressed using the following constraints:

$$
\sum_{\substack{h \in S \\ i \neq h}} f_{h i}-\sum_{\substack{h \in S \\ h \neq i}} f_{i h}+\sum_{j \in A} \sum_{k \in T_{j}} c_{j} y_{i j k}=0 \quad \forall i \in S \backslash S I N K
$$

$$
\sum_{j \in A} \sum_{k \in T_{j}} c_{j} z_{j}=\sum_{h \in S I N K}\left(\sum_{\substack{i \in S \\ i \neq h}} f_{i h}+\sum_{j \in A} \sum_{k \in T_{j}} c_{j} y_{h j k}\right)
$$

where $S I N K$ is the set of sink nodes (a subset of $S$ ) and $f_{i h}$ is a variable representing the flow of data in bps transmitted from node $i$ to node $h$. Constraints (7) enforce flow conservation at sensor nodes. Constraint (8) imposes that all the generated data are delivered to the set of sinks. The last term of this expression allows the sinks to be running applications as well.

The following constraint set enforces that if a sensor node is either running an application or receiving data, then it must be active in the network:

$$
\sum_{\substack{h \in S \\ h \neq i}} f_{h i}+\sum_{j \in A} \sum_{k \in T_{j}} c_{j} y_{i j k} \leq K x_{i} \quad \forall i \in S
$$

where $K$ is a constant high enough (higher than the maximum transmission rate of a node). Finally, constraints

$$
f_{i h} \leq K l_{i h} \quad \forall i, h \in S
$$

where $l_{i h}$ is a constant that indicates if there is a viable link between $i$ and $h$, i.e., if the distance between both nodes is less than the maximum transmission range $R_{\max }^{T}$, then $l_{i h}=1$ and $l_{i h}=0$ otherwise. Therefore, these constraints ensure that data must be transmitted exclusively along neighboring nodes.

These expressions allow flow splitting and multipath routing. However, in WSNs routes from each sensor node to a sink node follow typically a single path, such as the Destination Oriented Directed Acyclic Graph (DODAG) of RPL [22]. Therefore, we introduce the following restrictions to ensure that all the traffic flowing out of a sensor has only one possible route to a sink:

$$
\begin{array}{cc}
g_{i h} \leq l_{i h} & \forall i, h \in S \\
\sum_{h \in S} g_{i h} \leq 1 & \forall i \in S \\
f_{i h} \leq K g_{i h} & \forall i, h \in S
\end{array}
$$

where $g_{i h}$ is a binary variable which indicates if data are transmitted from node $i$ to node $h$. Constraints (12) and (13) impose that only one link from sensor node $i$ to any of its neighbors transports all the data that $i$ must forward.

The available bandwidth in the network is limited and must be shared among sensor nodes. We assume that a fair medium access control schemes orchestrate the access to the shared medium. Given a directional link between a pair of nodes $(i, h)$, let the capacity of the link be defined as $C_{i h}=$ $\min \left(C_{i}, C_{h}\right)$. This aims to model that the transmission rate is limited by the most restrictive node in the link. Transmissions of other links where $i$ or $h$ are either transmitter or receiver cannot be simultaneously active with $(i, h)$ (note that some combinations are not possible in this particular case due to routing constraints, i.e., another link with $i$ as a transmitter). 
According to the considered protocol interference model, the interfering links for link $(i, h)$ are those whose receiver is within the interference range of node $i$ or the links where $j$ is within the interference range of its transmitter. Although none of these links can be simultaneously active with $(i, h)$, some of them (depending on their relative positions) could be simultaneously active with each other. Therefore, if we define $I F_{i h}$ as the fraction of time that other links interfere the link $(i, h)$, we have that:

$$
\begin{array}{r}
I F_{i h}=\sum_{\substack{g \in S \\
g \neq h}} \frac{f_{i g}}{C_{i g}}+\sum_{g \in S} \frac{f_{g i}}{C_{g i}}+\sum_{\substack{g \in S \\
g \neq i}} \frac{f_{h g}}{C_{h g}}+\sum_{\substack{g \in S \\
g \neq i}} \frac{f_{g h}}{C_{g h}}+ \\
\sum_{\substack{g, t \in S \\
d_{i t}<R_{i}^{I}\left(p_{i}\right)}} \frac{f_{g t}}{C_{g t}}+\sum_{\substack{g, t \in S \\
d_{g h}<R_{g}^{I}\left(p_{g}\right)}} \frac{f_{g t}}{C_{g t}}
\end{array}
$$

Then, for each link $(i, h)$ in the network it must be ensured that the fraction of time used by the link plus all its interferences is less or equal to 1 :

$$
\frac{f_{i h}}{C_{i h}}+I F_{i h} \leq 1 \quad \forall i, h \in S
$$

Constraints (15) are the equivalent budget-type constraints for the available wireless capacity to the storage and processing load constraints given in (5) and (6).

Finally, energy constraints are included to ensure that the application deployment pattern guarantees a minimum lifetime $L$ for the virtual sensor network. Typically, energy consumption due to wireless communication (i.e., transmitting and receiving) has been considered the dominant factor in power consumption for WSNs [23]. While this is the case for traditional scalar applications, where processing is limited to simple operations, in multimedia applications the energy required to process data can not be neglected [24].

Regarding wireless transceiver, the power dissipation at the radio transmitter $P_{i}^{t}$ or at the radio receiver $P_{i}^{r}$ of each node $i$ can be modeled as [25]:

$$
\begin{gathered}
P_{i}^{t}=\sum_{h \in S, h \neq i}\left(\beta_{1}+\beta_{2} d_{i h}^{\gamma}\right) f_{i h} \quad \forall i \in S \\
P_{i}^{r}=\rho \sum_{h \in S, h \neq i} f_{h i} \quad \forall i \in S
\end{gathered}
$$

Typical values for $\beta_{1}, \beta_{2}$ and $\rho$ are $\beta_{1}=\rho=50 \mathrm{~nJ} / \mathrm{bit}$ and $\beta_{2}=0.0013 \mathrm{pJ} / \mathrm{bit} / \mathrm{m}^{4}$, with $\gamma=4$ the path loss index.

The estimation of the power dissipation due to the processing load is not so straightforward, since it depends on several factors such as the hardware architecture of the nodes or the specific implementations of the algorithms for each application. In the lifetime constraints set in (18), this power dissipation is left as a function $f$ of the proccessing loads $l_{j}$ of the applications. In Section IV, further details about the specific evaluated multimedia applications are given.

$$
P_{i}^{t}+P_{i}^{r}+f\left(\sum_{j \in A} \sum_{k \in T_{j}} y_{i j k} l_{j}\right) \leq \frac{E_{i}}{L} \quad \forall i \in S
$$

\section{Performance evaluation}

To evaluate the proposed model we have considered a scenario with two different types of sensor nodes and four different applications (two scalar and two multimedia). Next we define the main features of both sensor nodes and applications and the simulation parameters. Then, results are presented.

\section{A. Sensor nodes}

We have considered TelosB sensor motes [26] and BeagleBone nodes [27]. The energy budget for both nodes is $32400 \mathrm{~J}$ assuming that a node runs at $3 \mathrm{~V}$ with $3 \mathrm{Ah}$ of battery supply (2 AA batteries). Each TelosB mote has integrated a temperature and a light sensor, an IEEE 802.15.4 radio with integrated antenna and a $8 \mathrm{MHz}$ TI MSP430 microcontroller which can operate at 8 MIPS and with $10 \mathrm{~KB}$ RAM, although only $7 \mathrm{~KB}$ are available for applications [14]. Therefore, their resource vector is $o_{i}=\left\{C_{i}, M_{i}, L_{i}, E_{i}\right\}=$ $\{250 \mathrm{kbps}, 7 \mathrm{~KB}, 8 \mathrm{MIPS}, 32400 \mathrm{~J}\}$. These motes are suitable for supporting scalar applications. BeagleBone is a low-power platform based on a Linux Computer that includes $720 \mathrm{MHz}$ super-scalar ARM Cortex-A8 processor (up to 720 MIPS) and $256 \mathrm{MB}$ of RAM. BeagleBone nodes should include a Shimmer Span IEEE 802.15.4-compliant transceiver, a low-power USB camera for multimedia applications and also scalar sensors. Their resource vector is $o_{i}=\{250 \mathrm{kbps}, 256 \mathrm{MB}, 720 \mathrm{MIPS}, 32400 \mathrm{~J}\}$.

\section{B. Applications}

For the scalar applications, we have considered temperature and light monitoring. Temperature monitoring applications require 4462 bytes of RAM, while light monitoring applications require 1006 bytes [14]. This kind of applications has a low sample rate (0.017-1 Hz according to [28]). We have chosen a sample rate of $0.5 \mathrm{~Hz}$ for temperature monitoring and 1 $\mathrm{Hz}$ for light monitoring. Considering a packet size of 127 bytes per sample, the temperature application has a source rate of $0.5 \mathrm{kbps}$, whereas for the light application is $1 \mathrm{kbps}$. Given these parameters, we can assume that the processing load $l_{j}$ is negligible in the application requirement vector, (i.e. memory usage, transmission rate or the energy consumed by the transmission will be more limiting factors than the processing load or the energy consumed by the processing). Thus, the requirement vector for temperature monitoring is $r_{j}=\left\{c_{j}, m_{j}, l_{j}\right\}=\{0.5 \mathrm{kbps}, 4462 \mathrm{~B},-\}$ whereas for light monitoring is $r_{j}=\{1 \mathrm{kbps}, 1006 \mathrm{~B},-\}$

For multimedia applications we focus on visual sensor networks, i.e. WSNs designed to perform visual analysis (e.g. object recognition) [24]. We consider two paradigms, the classic Compress-Then-Analyze (CTA) and the opposite approach, Analyze-Then-Compress (ATC) [24], [29]. CTA applications are those where images acquired from camera 
nodes are compressed and sent to a central controller for further analysis. On the other hand, ATC applications are those where camera nodes perform visual feature extraction and transmit a compressed version of these features to a central controller. In [29] a detailed characterization of transmission rates and energy consumption for both approaches is provided. In order to evaluate the model with some realistic parameters, we have chosen some specific values for both cases based on the aforementioned study. It is assumed that different techniques for the extraction of local visual features are used: CTA will use the SIFT (Scale Invariant Feature Transform) algorithm while ATC will use BRISK (Binary Robust Invariant Scalable Keypoints) algorithm. Assuming a desired Mean of Average Precision (MAP) of 0.6, the use of Zurich Building Database $(\mathrm{ZuBuD})$ [30] and an application frame rate of $\lambda=$ 1 query per second for both CTA and ATC paradigms, the needed capacity will be $20 \mathrm{kbps}$ for CTA-SIFT and $12 \mathrm{kbps}$ for ATC-BRISK [29].

For this kind of applications, the energy consumed to process the data is not negligible. In [29] a characterization of this energy on a BeagleBone-based visual sensor node is provided. The processing energy for the CTA paradigm can be computed as:

$$
E_{c p u}^{C T A}(\rho)=P_{c p u} \cdot t_{c p u}^{C T A}(\rho)
$$

where $P_{c p u}$ is the power dissipated by the processor of the visual sensor node and has a value of $2.1 \mathrm{~W}$ for BeagleBone sensor nodes. $t_{c p u}^{C T A}(\rho)$ is the time spent for processing an image, which depends on $\rho$, the amount of sent information per query (20 kbs in our scenario). According to the results in [29], the processing energy for an image for the CTA application in our scenario is $0.05 \mathrm{~J}$. Therefore, assuming a frame rate of $\lambda=1$ query per second, the power dissipation (function $f$ in eq. (18)) is $0.05 \mathrm{~W}$. In addition, we can estimate the required processing load $l_{j}$ for a BeagleBone as the fraction of time used by the application $\left(t_{c p u}^{C T A} \cdot \lambda\right)$ multiplied by the processing power of the sensor node, $L_{i}$. In this case, $24.5 \cdot 1 \cdot 720=17.64$ MIPS.

Similarly, the processing energy for the ATC paradigm can be computed as:

$$
E_{c p u}^{A T C}(\rho)=P_{c p u} \cdot\left[\tau_{o f f}+M(\rho) \cdot\left(\tau_{\text {det }}+\tau_{\text {desc }}\right)\right]
$$

where $\tau_{o f f}$ is the time spent for initializing the detector and has a value of $1.6 \cdot 10^{-4} \mathrm{~ms} /$ pixel. With an image size of $640 \times 480$ pixels, $\tau_{\text {off }}$ is $49.152 \mathrm{~ms}$. $\tau_{\text {det }}$ and $\tau_{\text {desc }}$ are the time spent for detecting and describing one BRISK feature of the image and their values are $0.31 \mathrm{~ms}$ and $0.16 \mathrm{~ms}$ respectively. $M(\rho)$ is the optimal number of features that depends on the rate $\rho$. For $\rho=12 \mathrm{kbs} / q u e r y$, the minimum value of $M$ to provide a MAP of 0.6 is $M=100$ features. Thus the processing energy for an image for the ATC application in our scenario is $0.2 \mathrm{~J}$, and the power dissipation is $0.2 \mathrm{~W}$. The processing load in this case is 69.23 MIPS.

Regarding memory requirements, specific values have not been obtained for these applications. However, given the great difference in the amount of available memory in TelosB
(10 KB) and BeagleBone (256 MB), we are assuming that due to memory constraints, multimedia applications could not be implemented in TelosB nodes and memory will not be a limiting factor in BeagleBone nodes, since processing or transmission rate will limit long before these applications rather than memory. Summing up, the requirements vector for CTA and ATC applications are respectively $r_{j}=\left\{20 \mathrm{kbps}, 10 \mathrm{~KB}<m_{j}<<256 \mathrm{MB}, 17.64 \mathrm{MIPS}\right\}$ $r_{j}=\left\{12 \mathrm{kbps}, 10 \mathrm{~KB}<m_{j}<<256 \mathrm{MB}, 69.23 \mathrm{MIPS}\right\}$.

\section{Simulation Environment}

Sensor nodes are deployed in a 200×200 m scenario. We consider a default sensing range of $R_{i}^{s}=30 \mathrm{~m}$ for all of the sensors [31]. A two-ray ground path loss model with $\gamma=4$ and $g_{0}=8.1 \cdot 10^{-3}$ [32] is considered. $P_{\max }$ is set to $0 \mathrm{dBm}$ and the receiver sensitivity $\alpha$ is $-92 \mathrm{dBm}$ [33], which implies a maximum transmission range $R_{\max }^{T}$ of $59 \mathrm{~m}$. Analogously the interference sensitivity is $-104 \mathrm{dBm}$, which implies a maximum interference range $R_{\max }^{I}$ of $118 \mathrm{~m}$.

\section{Results}

As a reference example to evaluate the validity of the model and the benefits of virtualization we have considered a scenario with two different and overlapped WSNs: a scalar network, formed by 36 TelosB nodes and oriented to scalar applications (temperature and light monitoring), and a multimedia network, formed by 36 BeagleBone nodes and oriented to visual applications (CTA and ATC). The number of test points is 5 for the scalar applications and 3 for the visual ones. We assume that each sensor is able to cover $N_{i, j}=1$ test points of the same application and that each network have a sink node (one of the 36 nodes). The minimum lifetime for the virtual sensor network is $L=1$ day.

Figs. 1 and 2 show the performance of both networks in terms of number of active applications and required number of active nodes when the WSNs work isolated and also when the 72 nodes cooperate as a single network that gives support to all the applications. For each point in the curves, the same number of applications of each type (temperature, light, CTA and ATC) is generated, which is the value shown in the $x$-axes. For example, a 2 value in the $x$-axes represents a scenario where 2 temperature, 2 light, 2 CTA and 2 ATC applications try to be deployed. "Scalar" refers to the single scalar network, "Visual" to the single visual network and "Joint" to the cooperative whole network. In this latter case, two different preference vectors $Q$ are included: P1, where the preference is set to 1 for all the applications and P8, where the preference for the two visual applications is modified to 8.

Fig. 1(a) shows that the total number of active applications increases in the joint scenario, specially with P1 preference vector, when compared to the sum of the independent networks. As can be seen in Figs. 1(b) and 1(c), the main increase is due to scalar applications. In fact, when visual applications are not prioritized (P1), the increase in the number of scalar applications eventually leads to an starvation of 


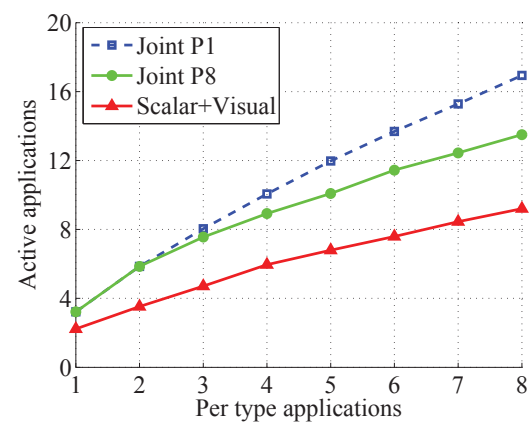

(a)

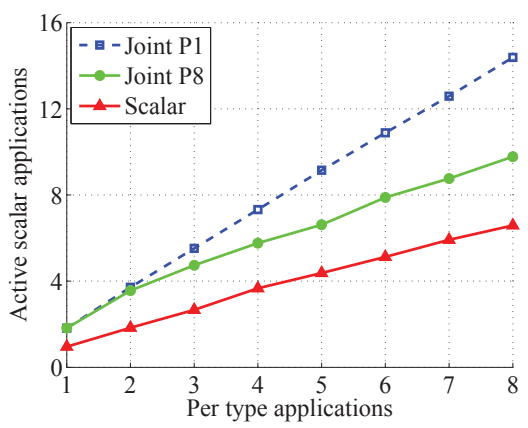

(b)

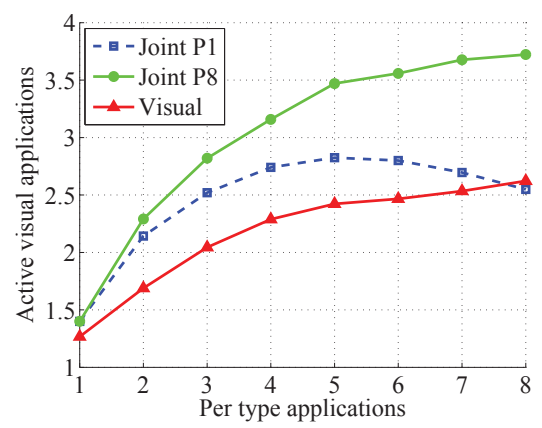

(c)

Fig. 1: Number of active applications vs. offered applications per type. a) Total b) Scalar c) Visual

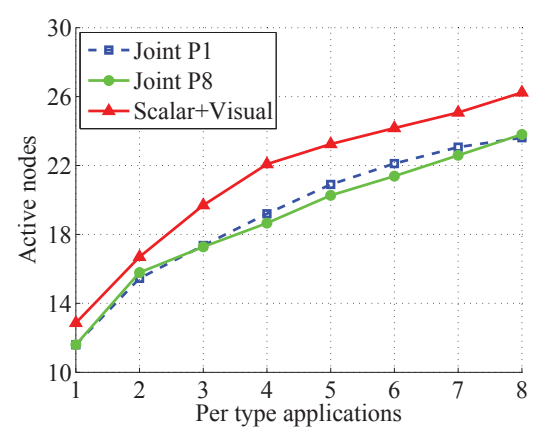

(a)

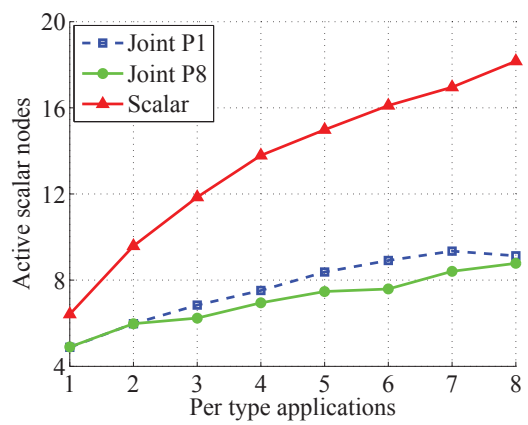

(b)

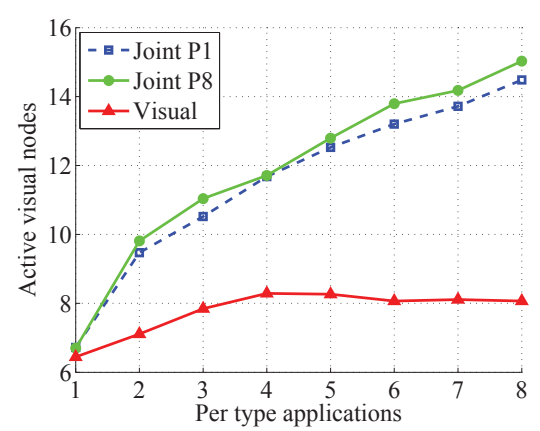

(c)

Fig. 2: Number of active nodes vs. offered applications per type. a) Total b) Scalar c) Visual

visual applications (Fig. 1(c)). However, with the preference vector P8 both scalar and visual applications experience an improvement.

The reasons for this improvement are different in each case: according to performed measurements, the probability of a test point not being covered with 36 nodes is about 0.15 . Since an active application requires all its test points to be effectively covered, the probability of a scalar application with 5 test points not being fully covered (only as topology concerns) is about 0.55 . As multimedia nodes can support scalar applications as well, in the joint scenario the 72 nodes can be used as sensors for scalar applications, reducing these probabilities to 0.015 and 0.07 respectively. Since scalar nodes cannot support multimedia applications, this kind of gain cannot be obtained for visual applications. Nevertheless, as one of the main limitations in multimedia networks is bandwidth (specifically the bottleneck in the transmission to the sink node), the possibility of using two sink nodes in the joint scenario leads also to an improvement for this case. Since the resources consumed by scalar applications is much lower than by visual ones, prioritizing the latter (P8) in the objective function is useful to balance the amount of resources used by each of them.

Finally, Fig. 2(a) shows that the total number of active nodes when both networks works jointly is lower than when they work isolated. Additionally, as the number of active applications is also higher, the active nodes per active application are quite lower when the networks work jointly. Regarding the type of active nodes, Fig. 2(c) shows that the amount of active multimedia nodes increases for the joint scenario. The reasons for this effect are two: (1) multimedia nodes can support scalar applications in the joint scenario and (2) we have assumed that the cost for activating sensor nodes, $\delta_{i}$, is the same for both kind of nodes. We have set $\delta_{i}=0.01$ to ensure that in any case the cost of activating the 72 nodes is higher than the revenue of activating an application.

\section{Conclusion}

In this paper we have analyzed a virtual sensor network where different kinds of applications and sensor nodes coexist and cooperate. We have formulated mathematically the optimization problem of maximizing the overall revenue out of the application deployment process while minimizing the cost related to activating sensor nodes. Constraints regarding sensor nodes capabilities (memory, computation, energy) and network limitations (topology, shared bandwidth) have been included. Realistic parameters for both the sensor nodes and the supported applications have been considered in the evaluation of the model. Simulation results have shown the potential 
performance gains that the resource reuse achieved by virtualization can obtain: coverage enhancements, since there is a higher density of sensor nodes capable of supporting a given application, and capacity increase, due to the possibility of reusing several sink nodes to reduce congestion on bottleneck links.

\section{ACKNOWLEDGMENT}

This work has been supported by the Spanish Government through the grant TEC2011-23037 from the Ministerio de Ciencia e Innovación (MICINN), Gobierno de Aragón (research group T98), the European Social Fund (ESF) and Centro Universitario de la Defensa through project CUD201305 .

\section{REFERENCES}

[1] A. Jayasumana, H. Qi, and T. Illangasekare, "Virtual sensor networks - a resource efficient approach for concurrent applications," in Proc. 4th International Conference on Information Technology (ITNG'07), Las Vegas, Apr. 2007, pp. 111-115.

[2] L. Sarakis, T. Zahariadis, H.-C. Leligou, and M. Dohler, "A framework for service provisioning in virtual sensor networks," EURASIP Journal on Wireless Communications and Networking, 2012.

[3] A. Merentitis, F. Zeiger, M. Huber, N. Frangiadakis, K. Mathioudakis, K. Sasloglo, G. Mazarakis, V. Gazis, and Z. Boufidis, "Wsn trends: Sensor infrastructure virtualization as a driver towards the evolution of the internet of things," in Proc. Seventh International Conference on Mobile Ubiquitous Computing, Systems, Services and Technologies UBICOMM 2013, Sep. 2013, pp. 113-118.

[4] M. K. Chowdhury and R. Boutaba, "A survey of network virtualization," Computer Networks, vol. 54, no. 5, pp. 862 - 876, apr 2010.

[5] Z. Xiao, W. Song, and Q. Chen, "Dynamic resource allocation using virtual machines for cloud computing environment," IEEE Transactions on Parallel and Distributed Systems, vol. 24, no. 6, pp. 1107 - 1117, jun 2013.

[6] M. M. Islam and E.-N. Huh, "Virtualization in wireless sensor network: Challenges and opportunities," Journal of Networks, vol. 7, no. 3, pp. 412 - 418, mar 2012

[7] A. Fischer, J. Botero, M. T. Beck, and H. de Meer, "Virtual network embedding: A survey," IEEE Communications Surveys and Tutorials, vol. 15, no. 4, pp. 1888 - 1906, 2013.

[8] M. M. Islam, M. M. Hassan, G.-W. Lee, and E.-N. Huh, “A survey on virtualization of wireless sensor networks," Sensors, vol. 12, pp. 2175 - 2207, 2012.

[9] P. Levis and D. Culler, "Mate: A tiny virtual machine for sensor networks," SIGARCH Comput. Archit. News, vol. 30, no. 5, pp. 85-95, Oct. 2002. [Online]. Available: http://doi.acm.org/10.1145/ 635506.605407

[10] P. Levis, D. Gay, and D. Culler, "Active sensor networks," in Proceedings of the 2Nd Conference on Symposium on Networked Systems Design \& Implementation - Volume 2, ser. NSDI'05. Berkeley, CA, USA: USENIX Association, 2005, pp. 343-356. [Online]. Available: http://dl.acm.org/citation.cfm?id=1251203.1251228

[11] Y. Yu, L. J. Rittle, V. Bhandari, and J. B. LeBrun, "Supporting concurrent applications in wireless sensor networks," in Proceedings of the 4th International Conference on Embedded Networked Sensor Systems, ser. SenSys '06. New York, NY, USA: ACM, 2006, pp. 139152. [Online]. Available: http://doi.acm.org/10.1145/1182807.1182822

[12] J. Koshy and R. Pandey, "Vmstar: Synthesizing scalable runtime environments for sensor networks," in Proceedings of the 3rd International Conference on Embedded Networked Sensor Systems, ser. SenSys '05. New York, NY, USA: ACM, 2005, pp. 243-254. [Online]. Available: http://doi.acm.org/10.1145/1098918.1098945

[13] I. Leontiadis, C. Efstratiou, C. Mascolo, and J. Crowcroft, "Senshare: Transforming sensor networks into multi-application sensing infrastructures," in Proceedings of the 9th European Conference on Wireless Sensor Networks, ser. EWSN'12, 2012, pp. 65-81.
[14] S. Bhattacharya, A. Saifullah, C. Lu, and G. Roman, "Multi-application deployment in shared sensor networks based on quality of monitoring," in Real-Time and Embedded Technology and Applications Symposium (RTAS), 2010 16th IEEE, April 2010, pp. 259-268.

[15] C.-L. Fok, C. Julien, G.-C. Roman, and C. Lu, "Challenges of satisfying multiple stakeholders: Quality of service in the internet of things," in Proceedings of the 2Nd Workshop on Software Engineering for Sensor Network Applications, ser. SESENA '11. New York, NY, USA: ACM, 2011, pp. 55-60. [Online]. Available: http://doi.acm.org/10.1145/1988051.1988062

[16] W. Li, F. C. Delicato, P. F. Pires, Y. C. Lee, A. Y. Zomaya, C. Miceli, and L. Pirmez, "Efficient allocation of resources in multiple heterogeneous wireless sensor networks," Journal of Parallel and Distributed Computing, vol. 74, no. 1, pp. 1775 - 1788, 2014. [Online]. Available: http://www.sciencedirect.com/science/article/pii/S0743731513002104

[17] Y. Xu, A. Saifullah, Y. Chen, C. Lu, and S. Bhattacharya, "Near optimal multi-application allocation in shared sensor networks," in Proceedings of the Eleventh ACM International Symposium on Mobile Ad Hoc Networking and Computing, ser. MobiHoc '10. New York, NY, USA: ACM, 2010, pp. 181-190. [Online]. Available: http://doi.acm.org/10.1145/1860093.1860118

[18] C. Wu, Y. Xu, Y. Chen, and C. Lu, "Submodular game for distributed application allocation in shared sensor networks," in INFOCOM, 2012 Proceedings IEEE, March 2012, pp. 127-135.

[19] C. de Farias, L. Pirmez, F. Delicato, W. Li, A. Zomaya, and J. De Souza, "A scheduling algorithm for shared sensor and actuator networks," in Information Networking (ICOIN), 2013 International Conference on, Jan 2013, pp. 648-653.

[20] N. Edalat, W. Xiao, M. Motani, N. Roy, and S. K. Das, "Auction-based task allocation with trust management for shared sensor networks," Security and Communication Networks, vol. 5, no. 11, pp. 1223-1234, 2012. [Online]. Available: http://dx.doi.org/10.1002/sec.631

[21] Y. Shi, T. Hou, J. Liu, and S. Kompella, "Bridging the gap between protocol and physical models for wireless networks," IEEE Transactions on Mobile Computing, vol. 12, no. 7, pp. 1404 - 1416, 2013.

[22] T. Winter, P. Thubert, A. Brandt, J. Hui, R. Kelsey, P. Levis, K. Pister, R. Struik, J. Vasseur, and R. Alexander, "RPL: IPv6 Routing Protocol for Low-Power and Lossy Networks,” RFC 6550 (Proposed Standard), Internet Engineering Task Force, Mar. 2012.

[23] I. Akyildiz, W. Su, Y. Sankarasubramaniam, and E. Cayirci, "Wireless sensor networks: A survey," Computer Networks, vol. 38, no. 4, pp. 393 $-422,2002$.

[24] A. Redondi, M. Tagliasacchi, and M. Cesana, "Rate-accuracy optimization in visual wireless sensor networks," in IEEE International Conference on Image Processing (ICIP2012), Orlando, Florida, Oct. 2012, pp. 1105-1108.

[25] T. Hou, Y. Shi, and H. Sherali, "Rate allocation and network lifetime problems for wireless sensor networks," IEEE/ACM Transactions on Networking, vol. 16, no. 2, pp. 321 - 334, 2008.

[26] TelosB Mote Platform Datasheet, MEMSIC Inc.

[27] G. Coley, Beaglebone rev a6 system reference manual, 2012.

[28] J. B. Javier Molina, Javier M. Mora-merchan and C. Leon, Wireless Sensor Networks: Application. InTech, 2010, ch. Multimedia Data Processing and Delivery in Wireless Sensor Networks.

[29] A. E. Redondi, "Energy-aware visual analysis for wireless multimedia sensor networks," Ph.D. dissertation, Politecnico Di Milano, 2014.

[30] H. Shao, T. Svoboda, and L. V. Gool, "Zubud zurich buildings database for image based recognition," Tech. Rep. 260, March 2003, computer Vision Laboratory, Swiss Federal Institute of Technology.

[31] A. Chen, S. Kumar, and T. H. Lai, "Designing localized algorithms for barrier coverage," in Proceedings of the 13th Annual ACM International Conference on Mobile Computing and Networking, ser. MobiCom '07. New York, NY, USA: ACM, 2007, pp. 63-74.

[32] C. Suh, J.-E. Joung, and Y.-B. Ko, "New rf models of the tinyos simulator for ieee 802.15.4 standard," in Proc. IEEE WCNC 2007, Hong Kong, march 2007, pp. 2238-2242.

[33] CC2420: "Datasheet for Chipcon (TI) CC2420 2.4 GHz IEEE 802.15.4/ZigBee RF Transceiver". 